\title{
LES MICROÉMULSIONS RELÈVENT-ELLES DES PHÉNOMÈNES POLYCRITIQUES ?
}

\author{
A. SKOULIOS et D. GUILLON \\ Centre de Recherches sur les Macromolécules, 6, rue Boussingault, 67083 Strasbourg, France
}

(Reçu le 14 décembre 1976, accepté le 3 février 1977)

\begin{abstract}
Résumé. - La formation des micelles dans les systèmes ternaires amphiphile/eau/huile et leurs changements de forme ont été considérés comme étant des transitions de type continu du second ordre. La question s'est alors posée de savoir si la formation des microémulsions ne relèverait pas des phénomènes polycritiques, étant donné la possibilité de rencontre entre les lignes de transitions micellaires et osmotique de démixtion entre phases aqueuse et huileuse.
\end{abstract}

\begin{abstract}
The formation of micelles in the ternary amphiphile/water/oil systems and their shape modifications have been considered as being similar to continuous second-order transitions. The question then arises as to how far the formation of microemulsions can be considered as polycritical phenomena; the micellar transition lines and the first order demixtion line of oil and water phases may very well intersect to form high-order critical points.
\end{abstract}

Observées pour la première fois en 1943 [1], les microémulsions suscitent à l'heure actuelle un intérêt considérable, tant théorique que pratique $[2,3,4]$. Avant de les définir et d'en décrire les propriétés essentielles, il est bon de rappeler brièvement les caractéristiques de deux autres systèmes apparentés : les émulsions et les solutions micellaires.

Les émulsions [5] sont des systèmes hétérogènes, formés de deux liquides non miscibles, dont l'un est finement dispersé dans l'autre sous forme de gouttelettes de quelques centaines, voire de quelques milliers de nanomètres de diamètre. On peut citer en exemple les émulsions d'huile dans l'eau, ou d'eau dans l'huile. Par suite de la tension interfaciale positive entre les deux liquides, les émulsions ne sont pas stables mais décantent spontanément en deux phases superposées. En présence d'un agent tensio-actif (amphiphile : savon, ...), dont les molécules se concentrent aux interfaces, la tension interfaciale devient plus faible et la décantation du système s'opère plus lentement (d'où la stabil'té apparente de certaines émulsions). Dans un diagramme de phases (Fig. 1), la zone d'existence des émulsions est schématiquement limitée par une courbe de démixtion $\mathrm{ABC}$ : une émulsion de composition $M$ se séparera à la longue en deux phases macroscopiques, l'une aqueuse, l'autre huileuse, de compositions respectives $M_{1}$ et $M_{2}$.

Les solutions micellaires [6] ont été observées dans les mélanges binaires d'eau et de savon. Lorsque la concentration est très faible, le savon est moléculairement dispersé dans le milieu. En revanche, lorsque la concentration est plus grande, les molécules de savon

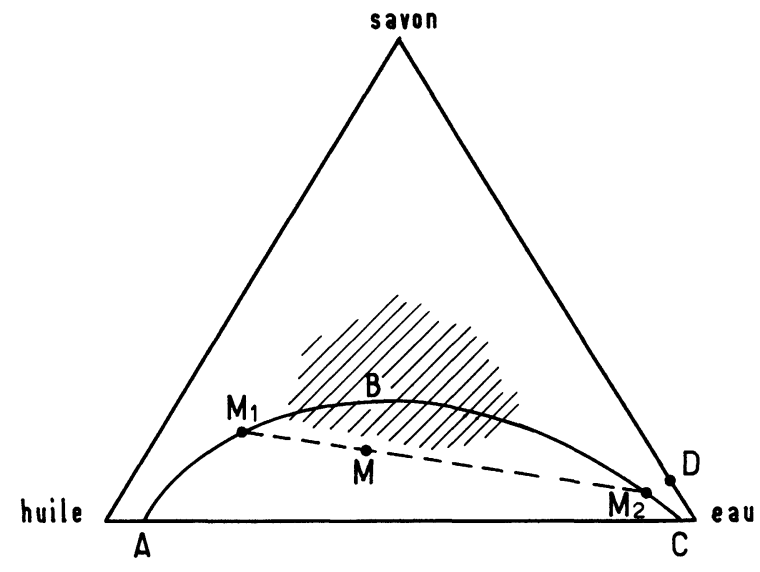

FIG. 1. - Schéma de diagramme de phases.

[Sketch of the phase diagram.]

se groupent en micelles. Celles-ci sont formées par les chaînes aliphatiques désordonnées et sont recouvertes par les groupes polaires des molécules de savon; elles ont typiquement un diamètre de quelques nanomètres. Le passage de la solution moléculaire à la solution micellaire s'effectue dans une zone étroite de concentrations, appelée concentration micellaire critique : $\mathrm{cmc}$ (point $\mathrm{D}$ dans la figure 1). Généralement, les micelles ont une forme sphérique; il est cependant fréquent de les rencontrer sous la forme allongée de bâtonnets, voire même aplatie de disques. La transition entre ces formes s'effectue apparemment de façon continue mass rapide en fonction de la concen- 
tration ou de la température [7, 8]. Des micelles inverses ont de même été observées dans des milieux non aqueux; les groupes polaires des molécules de savon forment alors le cœur des micelles, les chaînes aliphatiques étant rejetées vers l'extérieur. Longtemps controversée, leur existence semble maintenant admise [9] sans difficultés.

La solubilité d'huile dans les solutions très diluées de savon dans l'eau est extrêmement faible; elle augmente de manière spectaculaire à la $\mathrm{cmc}$. Ce phénomène est dû à la possibilité de l'huile à se localiser au cœur des micelles, qui est constitué par les chaînes aliphatiques désordonnées des molécules de savon. Un phénomène symétrique, concernant la solubilisation d'eau, peut être envisagé dans le cas des micelles inverses.

La formation des micelles, du moins dans les solutions aqueuses, a été considérée jusqu'ici, soit comme un processus d'association par étapes des molécules de savon (une cascade d'équilibres entre micelles de tailles différentes, régis par la loi d'action de masse), soit comme un phénomène de démixtion (à la $\mathrm{cmc}$, apparaissent des micelles en démixtion avec la solution moléculaire) $[10,11,12]$, soit encore tout récemment comme la formation de défauts bidimensionnels [13]. De ces points de vue, le deuxième est de plus en plus contesté, car, dans la région de la $\mathrm{cmc}$, les propriétés physicochimiques varient rapidement, mais sans présenter de discontinuité, avec la concentration, et, dans la région micellaire, elles continuent à évoluer de manière sensible. Tel est notamment le cas de la pression osmotique, de la densité, ou de la diffusion de la lumière. Bien qu'à notre connaissance le phénomène de micellisation n'ait pas encore été considéré sous cet angle, il s'apparente à notre avis singulièrement à une transition continue du second ordre. Il reste, certes, de nombreux points à éclaircir avant d'admettre que tel est effectivement le cas ; néanmoins, à partir du moment où toutes les manifestations macroscopiques de la formation des micelles sont celles d'une transition continue, nous pensons qu'il est intéressant de rechercher les conséquences de cette conception dans l'analyse et la compréhension des mélanges savon/eau/huile.

Les microémulsions [3] sont à maints égards des systèmes intermédiaires entre les émulsions et les solutions micellaires gonflées de solvant. Apparemment stables et macroscopiquement homogènes et transparentes, elles sont formées d'une dispersion très fine d'huile dans l'eau, ou d'eau dans l'huile ; typiquement, la dimension des gouttelettes qu'elles renferment est de quelques dizaines de nanomètres. Généralement, encore que ce ne soit pas systématique, elles sont obtenues par addition à une émulsion d'un agent tensio-actif auxiliaire (cosurfactant), tel un alcool gras par exemple; des mesures de diffusion de la lumière faites dans certains cas montrent que la dimension des gouttelettes passe par un minimum en fonction de la concentration en cosurfactant [14].
Leur viscosité est le plus souvent faible; on cite [3] cependant des cas où la viscosité augmente considérablement à l'approche de la transformation de la microémulsion en émulsion ordinaire. Suivant la proportion relative de l'eau et de l'huile, la température, la balance hydrophile/lipophile de l'agent tensio-actif et du cosurfactant, ainsi que l'affinité de l'huile à l'égard de l'eau, on peut observer des microémulsions de type eau dans l'huile, ou huile dans l'eau. $\mathrm{Du}$ point de vue de leur composition chimique, les microémulsions se situent dans le diagramme de phases au-delà de la zone d'existence de l'émulsion (région hachurée ; Fig. 1).

Pour expliquer la formation spontanée des microémulsions, on invoque [15] le plus souvent la notion de tension interfaciale négative. On constate, il est vrai, que cette tension s'abaisse considérablement par addition de cosurfactant jusqu'à s'annuler même à l'approche de la région d'existence des microémulsions. Cette notion est toutefois contestable du strict point de vue thermodynamique (voir [16], page 549).

Après avoir défini d'une manière aussi simple que possible la nature des microémulsions et leurs propriétés fondamentales, et après avoir dégagé leurs similitudes et leurs différences avec les émulsions et les solutions micellaires, nous allons essayer d'analyser leur formation en nous aidant de ce que l'on sait des phénomènes critiques [17].

Revenons pour cela au diagramme de phases du système savon/eau/huile que, pour simplifier, nous supposerons ternaire, le savon comprenant une quantité variable de cosurfactant. Par addition d'huile au mélange savon/eau, la cmc décrira dans le diagramme une ligne (DE) qui rencontre la ligne $\mathrm{ABC}$ de démixtion au point $E$ (Fig. 2). Ce point représente un point singulier, qui sera un point critique d'ordre supérieur puisque la ligne de transition micellaire est une ligne de transition continue du second or̀dre $[17,18]$.

Une situation analogue se produit également dans le cas du mélange binaire ${ }^{3} \mathrm{He} /{ }^{4} \mathrm{He}$ à très basse tem-

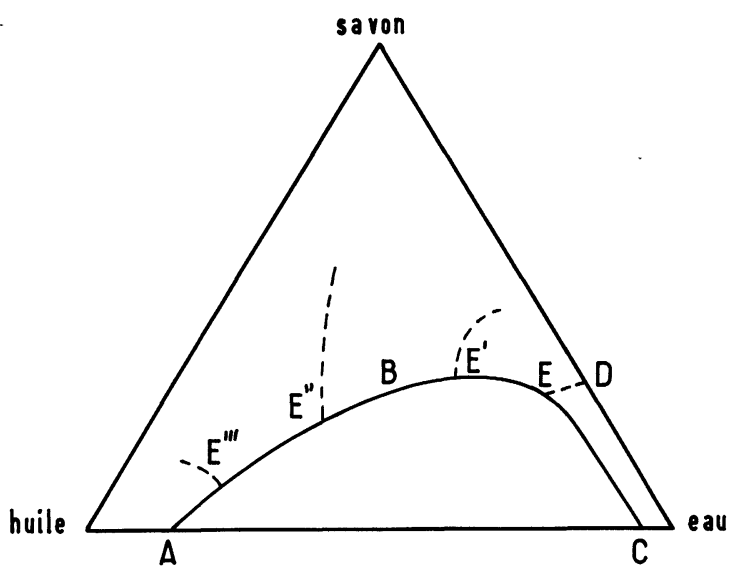

Fig. 2. - Représentation schématique de l'intersection de lignes de transition continue avec la ligne de démixtion.

[Schematic representation of the intersection with the demixtion line of the lines of continuous transitions.] 
pérature $(1 \mathrm{~K})$. Dans ce système, la ligne de transition superfluide, qui est une transition continue du second ordre, vient couper la ligne de transition de premier ordre, qui est une transition de démixtion entre deux phases liquides, inégalement riches en ${ }^{3} \mathrm{He}$. Le point d'intersection est décrit comme étant un point tricritique [18].

Dans le voisinage d'un point tricritique, le régime du système est gouverné par la compétition de deux paramètres d'ordre : dans le cas de l'hélium par exemple, par la compétition des paramètres d'ordre superfluide et osmotique de démixtion. Dans le cas des mélanges savon/eau/huile, le régime du système fait intervenir la compétition entre les paramètres d'ordre micellaire et osmotique de démixtion.

Si tel s'avère être le cas, on peut avancer pour la formation des microémulsions l'hypothèse suivante. Par suite de la compétition entre les paramètres d'ordre micellaire et osmotique, le système hésite au gré des fluctuations locales entre la structure micellaire et la structure d'émulsion. Si, d'une part, le temps nécessaire à la coalescence de l'émulsion et à sa décantation macroscopique dépasse largement le temps de corrélation des fluctuations osmotiques, et si, d'autre part, le temps nécessaire à la formation des micelles n'est pas excessif par rapport au temps de corrélation des fluctuations micellaires, on conçoit aisément que le système puisse se comporter, en se renouvelant sans cesse, comme s'il était stable. Cette stabilité est de nature éminemment dynamique. On conçoit également que les degrés d'avancement des processus de coalescence de l'émulsion et de micellisation, mesurés à l'échelle des temps de corrélation correspondants, puissent déterminer la polydispersité et la finesse moyenne des microémulsions. Sachant que la vitesse de coalescence des émulsions est très petite (par suite notamment des tensions interfaciales faibles) et la vitesse de formation des micelles généralement grande, on conçoit sans difficultés que le régime critique exerce une influence encore sensible loin du point tricritique, c'est-à-dire dans une zone assez étendue dans le diagramme de phases.

Au stade actuel de nos connaissances, notamment en ce qui concerne la nature exacte des transitions micellaires, il serait prématuré de rechercher à préciser l'ordre du phénomène polycritique invoqué et à caractériser ses paramètres. On ignore en effet si d'autres transitions micellaires que celles dont nous venons de parler, telles par exemple les transitions de formation de micelles inverses ou de changement de forme, ne sont pas susceptibles d'intervenir également. Ces transitions pourraient expliquer de façon satisfaisante la localisation centrale des microémulsions dans le diagramme de phases (voir points polycritiques $E^{\prime}, E^{\prime \prime} . . .$, Fig. 2) et leur inversion. De plus, la présence dans le diagramme de phases d'une multitude de régions où existent et coexistent des phases mésomorphes lyotropes nombreuses et variées vient compliquer encore davantage la situation et nous invite à une très grande prudence.

Les champs susceptibles de jouer un rôle pertinent dans les phénomènes que nous venons de décrire sont nombreux. On peut penser par exemple aux potentiels chimiques de l'eau et de l'huile, ou à la température. On peut aussi penser à un champ lié à l'activité du cosurfactant. Ce dernier n'est pas forcément le potentiel chimique du cosurfactant luimême ; il doit simplement tenir compte de la contribution du cosurfactant à l'activité de surface du savon auquel il est associé.

Sans préjuger des conclusions auxquelles pourra conduire une étude approfondie du système, tant sur le plan théorique (existence de transitions du second ordre dans les solutions micellaires, rôle de la symétrie, définition des paramètres d'ordre, etc.) qu'expérimental (évolution de la cmc avec la teneur en huile, etc.), on peut penser qu'aborder le problème des colloïdes d'association dans l'optique des phénomènes critiques pourra servir à une meilleure compréhension de leur comportement et à une classification plus claire des mésophases lyotropes, des solutions colloïdales et des émulsions.

Remerciements. - Nous tenons à remercier MM. M. Papoular, F. Gautier, R. Zana et H. Benoît pour les discussions extrêmement fructueuses que nous avons eues, ainsi que M. P. G. de Gennes pour nous avoir rendus attentifs aux difficultés théoriques inhérentes aux transitions micellaires.

Note. - Ce travail repose sur l'idée que le phénomène de micellisation est une transition continue du second ordre. Cette affirmation a été discutée par nos rapporteurs. Elle peut être mise en doute actuellement, mais elle conduit les auteurs à proposer une approche nouvelle, que nous avons jugée stimulante, du difficile problème de la formation des microémulsions.

\section{Bibliographie}

[1] Hoar, T. P. et Schulman, J. H., Nature 152 (1943) 102.

[2] Shinoda, K. et Friberg, S., Adv. Coll. Interf. Sci. 4 (1975) 281.

[3] Bavière, M., Revue de l'IFP, éd. Technip, Paris 29 (1974) 41.

[4] Shinoda, K. et Kunieda, H., J. Coll. Interf. Sci. 42 (1973) 381 .
[5] Becher, P., Emulsions (Rheinh. Publ. Corp., NY) 1966.

[6] Lire par exemple : MUKERJEe, P., in Physical Chemistry : enriching topics from colloid and surface science, IUPAC Commission I.6, éd. H. van Olphen et K. J. Mysels, Theorex, La Jolla, Calif. (1975). 
[7] Corkill, J. M. et Herrmann, K. W., J. Phys. Chem. 67 (1963) 934.

[8] Reiss-Husson, F. et Luzzati, V., J. Phys. Chem. 68 (1964) 3504.

[9] Pethica, B., Proc. Intern. Congr. Surf. Activity, Cologne 1 (1960) 212.

[10] Shinoda, K., Nakagawa, T., Tamamushi, B. et Isemura, T., Colloidal Surfactants (Acad. Press, NY) 1963.

[11] Mukerjee, P., Adv. Coll. Interf. Sci. 1 (1967) 241.

[12] Ruckenstein, E. et Nagarajan, R., J. Phys. Chem. 79 (1975) 2622.
[13] Parodi, O., J. Physique Lett. 37 (1976) L-295.

[14] Prince, L. M., J. Coll. Interf. Sci. 23 (1967) 165.

[15] Schulman, J. H. et Montagne, J. B., Ann. N. Y. Acad. Sci. 92 (1961) 366.

[16] Landau, L. et Lifchitz, E., Physique statistique (éd. Mir, Moscou) 1957.

[17] Toulouse, G. et Pfeuty, P., Introduction au groupe de renormalisation et à ses applications (Presses Universitaires de Grenoble) 1975.

[18] Papoular, M., J. Physique Colloq. 37 (1976) C1-237. 